\title{
Mindful eating verses mindless eating
}

\section{Introduction}

As the growing fast food culture in our society, we don't have enough time to sit properly for our main and mid meals. It's too common to hear of people, grabbing breakfast on the run or attending a lunch meeting. The speed of eating isn't the only problem, eating while multitasking such as working during lunch, watching television or driving a car is actually resulting in the consumption of large number of food unconsciously but not feels full. Without self-awareness, people eat without regard to the quality and quantity of food that passing their lips. The whole phenomenon is called MINDLESSS eating or eating without awareness. This mindless behavior can have many negative health consequences and Obesity is one of them. ${ }^{1,2}$

There is also a need to understand the precise mechanism of hunger and fullness and the signals of our central nervous system, when food is desired or needed. Eating too quickly and mindlessly also disturbed the communication between gut and brain which can cause many GI problems like gas and bloating. ${ }^{3-5}$

Mindfulness is actually the moment to moment awareness of our thoughts, feelings and surroundings. It is a non- diet approach to achieve and maintain healthy weight with long term benefits. MINDFULL EATING means "Eat with attention". It involves developing and enjoyable relationship with food. It's not any kind of restrictive diet but instead, this practice involves experiencing the pleasure of food more intensely and being fully present at the meal. It promotes a psychological barrier to overeating and emotional eating by paying attention to the body signals such as hunger, fullness and satisfaction. ${ }^{6,7}$

Many researchers are working on teaching obese individuals; mind full eating skills which can help them change their unhealthy eating patterns and lose weight. It is also helping people to make them aware about their physical and emotional cues around eating and to learn to savor their food more. ${ }^{5}$

Mindful eating techniques are actually based on BuddhistPhilosophy. Mindful eating can be thought of as a type of meditation. Researchers said that Eating more slowly and relishing each bite could help people eat less and healthier. These techniques exist to help us to live in "Present Moment" while we eat and enjoy the benefits of our meals in terms of satisfying our hunger, taste buds and nutritional needs. Some practices to try include the following. ${ }^{8}$

a. Take a small bite of food, serve only the amount of food you think you will need.

b. Close your eyes and chew it thoroughly(20-30 min) in a silent atmosphere

c. Enjoy every bite, while paying attention to texture, taste and temperature of the food.

d. Eat without any distraction, without watching television or any ideas running through your mind.

e. Take a deep breath and exhale after finishing your every bite

f. Express gratitude and appreciation that you are able to taste something so delightful
Volume 4 Issue 3 - 2016

\author{
Maria Nadeem \\ University of the Punjab, Pakistan
}

Correspondence: Maria Nadeem, Clinical Nutritionist and Dietician, University of the Punjab, 388-Rblock, Johar Town Lahore, Pakistan, Tel 0321482224I,

Email marianadeem2007@hotmail.com

Received: December 17, 2015 | Published: March 16, 2016

\section{g. Stop eating when no longer hungry ${ }^{5,7}$}

Try to do this practice twice a day and more than three days a week. It's too easy to learn and it swiftly gets better and easier with practice. There is much to be said about the benefits of mindful eating, but in short, it develops a positive relationship between food and eating leading towards a healthy, contented and stress less life. ${ }^{6,4}$

\section{Acknowledgements}

None.

\section{Conflict of interest}

The author declares no conflict of interest.

\section{References}

1. Lillis J, Hayes SC, Bunting K, et al. Teaching acceptance and mindfulness to improve the lives of the obese: a preliminary test of a theoretical model. Ann Behav Med. 2009;37(1):58-69.

2. Tapper K, Shaw C, Ilsley J, et al. Exploratory randomised controlled trial of a mindfulness based weight loss intervention for women. Appetite. 2009;52(2):396-404.

3. http://greatergood.berkeley.edu/article/item/better_eating_through_ mindfulness

4. http://www.medicaldaily.com/obesity-rate-may-improve-mindfuleating-how-meditating-during-meals-helps-weight-loss-358866

5. http://www.obesityaction.org/educational-resources/resource-articles-2/ nutrition/love-what-you-eat-how-mindful-eating-helps-break-your-eatrepent-repeat-cycle

6. Singh NN, Lancioni GE, Ashvind NS, et al. A mindfulness-based health wellness program for managing morbid obesity. Clinical Case Studies. 2008;7(4):327-339.

7. Jennifer D, Jean K, Frederick MH, et al. Mindfulness Intervention for Stress Eating to Reduce Cortisol and Abdominal Fat among Overweight and Obese Women: An Exploratory Randomized Controlled Study. Journal of Obesity. 2011;2011(2011):1-13.

8. Smith BW, Shelley BM, Leahigh L, et al. A preliminary Study of the Effects of a Modified Mindfulness Intervention on Binge Eating. Journal of Evidence-Based Complimentary \& Alternative Medicine. 2006;11(3):133-143. 